\title{
Ice gouge depth determination via an efficient stochastic dynamics technique
}

\author{
Nikolaos Gazis \\ Wood Group, \\ 15115 Park Row \\ Houston, TX 77019, USA \\ nikolaos.gazis@woodgroup.com \\ loannis A. Kougioumtzoglou \\ Department of Civil Engineering \& Engineering Mechanics, Columbia University, \\ 500 W. 120th St., New York, NY 10027, USA \\ ikougioum@columbia.edu

\section{Edoardo Patelli} \\ Institute for Risk \& Uncertainty, University of Liverpool, \\ Liverpool L69 3BX, United Kingdom \\ edoardo.patelli@liverpool.ac.uk
}

\begin{abstract}
A simplified model of the motion of a grounding iceberg for determining the gouge depth into the seabed is proposed. Specifically, taking into account uncertainties relating to the soil strength, a nonlinear stochastic differential equation governing the evolution of the gouge length/depth in time is derived. Further, a recently developed Wiener path integral based approach for solving approximately the nonlinear stochastic differential equation is employed; thus, circumventing computationally demanding Monte Carlo based simulations, and rendering the approach potentially useful for preliminary design applications. The accuracy/reliability of the approach is demonstrated via comparisons with pertinent Monte Carlo simulation data.
\end{abstract}




\section{INTRODUCTION}

Oil and gas (O\&G) operators have been focusing their efforts on exploration and development of arctic regions the last several years as traditional fields are rapidly depleting [1]. It has been estimated that $22 \%$ of the world's undiscovered reserves are located in the arctic circle, $84 \%$ of which are located offshore [2]. One main concern with offshore oil and gas development in the arctic is seabed scouring due to iceberg impact with the soil (ice gouging). Offshore pipelines in the arctic are buried below the mud line so as to be protected from iceberg impact. The burial process involves trenching the seabed before laying the pipeline. However, trenching costs increase significantly with burial depth, potentially even exceeding the cost of the pipeline fabrication itself [3]. Therefore, a sustained challenge in the O\&G industry is the accurate prediction of the depth of the ice gouge, and consequent pipeline embedment design depth.

Seabed surveys can be used to directly measure the gouge depth. However, measurements of existing berg induced seabed scours may not accurately predict the potential future gouge event as infilling of the scour trenches occurs. Further, it is difficult to determine if the observed scours are recent or relics. Another approach is to conduct multiple seabed surveys over several years to determine scouring rates relative to time and space. However, this requires a tremendous amount of resources and is not feasible due to the high associated costs. Therefore, it is important to understand the mechanics of the grounding berg to predict realistic gouge depths using theoretical models. 
Sophisticated finite element method (FEM) based models have been developed to predict the ice gouge behavior and the effects of ice gouging on buried pipelines [410]. In [11] a FEM based model was employed in a parametric study to investigate the effect of different physical factors on gouge depth. A semi-empirical relationship, which can be construed as a "meta-model", was derived to estimate gouge depth and utilized in a probabilistic analysis within a Monte Carlo simulation (MCS) context. FEM based approaches require significantly more computational effort when compared to more analytical approaches. Additionally, it can be argued that the overall accuracy of such detailed FEM approaches is not necessarily higher than that of more approximate analytical approaches. This is due to the high level of uncertainty involved in the selection of parameter values related to the system (e.g. iceberg shape/weight etc.) and to the excitation/environment (e.g. sea current characteristics). Clearly, because of this apparent inconsistency between a very detailed FEM modeling and a high degree of uncertainty regarding the involved parameter values, the desired outcome of overall enhanced accuracy is at least a debatable one. Furthermore, even if the effects of uncertainties were considered in these elaborate FEM models in a comprehensive manner via an appropriate stochastic modeling, determining the system stochastic response using brute force MCS based approaches would be, potentially, computationally prohibitive $[12,13]$. A comprehensive discussion of some of the challenges in FEM based approaches is presented in [14].

In this regard, several researchers have proposed approximate analytical treatments of the ice-gouging problem. In [15] a model was developed that equates the 
kinetic energy of the moving berg to the work done in plowing into the soil. The energy is computed considering the mass of the berg and the environmental factors (sea current and current drag) while the soil resistance is determined based on a passive pressure mechanism at the face of the grounding berg; see also $[16,17]$. Further, $[18,19]$ considered the soil deformation ahead of the berg keel, assuming two-dimensional plane strain and relying on plasticity theory. In [20] a model of the grounding berg in sandy soils was developed based on experimental work that incorporates passive soil resistance on the front and sides of the berg and sliding resistance on the bottom. Reference [21] used a differential equation that balances the forces of inertia, current drag and soil resistance to compute the gouge length. A comprehensive presentation of the various theoretical ice gouge models can be found in $[3,22]$.

The current work extends and generalizes the model proposed in [21] circumventing some of its limitations. Specifically, first, the model proposed in [21] exhibits an oscillatory response behavior, which, clearly, is not physically realistic for the ice-gouging problem. Therefore, an energy dissipation term is added to the model that prevents oscillatory behavior. The result can be construed as an "over-critically damped" nonlinear dynamical system (e.g. [23]).

Second, most of the theoretical models in the literature (including the model in [21]) are deterministic, and thus, it can be argued that they cannot capture many aspects of the ice gouge mechanism as the uncertainties inherent in the environment are not considered. Therefore, a natural extension/generalization to the proposed gouge model is to consider the variations in the environmental parameters that affect 
the response. In this regard, a stochastic treatment of the proposed dynamical system is considered by taking into account uncertainties related to the soil strength; this yields a second-order nonlinear stochastic differential equation (SDE) governing the evolution in time of the gouge length. Next, the recently developed Wiener path integral (WPI) technique for treating certain random vibration problems (e.g. $[24,25])$ is applied to efficiently solve the SDE governing the ice gouging motion; thus, computationally demanding MCS are circumvented. Specifically, a variational formulation is utilized to derive an Euler-Lagrange (E-L) equation governing the "most probable path". The resulting boundary value problem (BVP) is then solved numerically and the response probability density function (PDF) for the gouge depth at a given point is obtained. Further, the accuracy of the WPI based solution approach is demonstrated by comparing the results to pertinent MCS data.

The model utilized in the proposed approach is a simplification of the gouging phenomenon, however, it requires significantly less computational time when compared to previously developed FEM based approaches. Further, as discussed in [3], FEM based models require rigorous validation against quality data from physical simulations. Furthermore, conducting a probabilistic analysis utilizing a FEM based model in a MCS can be computationally prohibitive. The WPI technique adopted in the proposed approach is orders of magnitude less computationally demanding when compared to brute force MCS based approaches. The combination of a simplified model and the efficient WPI based solution establishes the proposed approach as a viable alternative to previous approaches, at least at a preliminary design level. 


\section{ICE GOUGE MODEL}

The formulation in [21] is delineated in the following subsection followed by the proposed enhancement to the model. A numerical example is presented comparing the two models and the results are discussed. Next, modeling the soil strength as a stochastic process further extends the enhanced formulation governing the ice gouging. This leads to a nonlinear second order stochastic differential equation governing the berg dynamics.

\section{Equation of Motion Governing Ice Gouging}

The berg is assumed to be freely drifting initially with a velocity equal to that of the propelling currents averaged over the immersed depth of the berg. As shallower water depths are reached the berg will tend to ground in the course of its travel. The forces acting on the berg during gouging process are the hydrodynamic force from the current, the soil resistance and the inertial force of the berg. Balancing the forces yields the following expression [21], $m \ddot{x}+P_{s}=f(t)$

where $m$ is the mass of the berg, $P_{s}$ is the of soil resistance force, $f(t)$ is the hydrodynamic force and $x$ is the gouge length. The most widely used expression for a hydrodynamic force is based upon Morison's equation [26],

$f(t)=\rho V_{b} \dot{v}+\left(C_{m}-1\right) \rho V_{b}(\dot{v}-\ddot{x})+\frac{1}{2} C_{d} \rho A_{b}|v-\dot{x}|(v-\dot{x})$

where $\rho$ is the density of seawater, $V_{b}$ is the volume of the berg, $A_{b}$ is the crosssectional area of the berg normal to the drag force, $C_{m}$ and $C_{d}$ are the mass and drag 
coefficients, respectively, and $v$ is the velocity of the current. Since the current velocity is assumed to be constant, Eq. 2 reduces to

$f(t)=\left(C_{m}-1\right) \rho V_{b} \ddot{x}+\frac{1}{2} C_{d} \rho A_{b}|v-\dot{x}|(v-\dot{x})$.

The soil resistance force, $P_{S}$, is approximated as

$\mathrm{P}_{s}=\mathrm{K}_{\mathrm{s}} \mathrm{d}^{2}$

where $K_{S}$ is the coefficient of soil resistance and $d$ is the gouge depth (see also [21]). Note that Eq. 4 depicts the force required to push a smooth vertical wall into cohesionless soil and is based on Coulomb's passive earth pressure theory [27]. It has been shown in plowing force estimation studies (e.g. [28]) that Eq. 4 underestimates the plowing force as it ignores the shearing of the sidewalls and bottom surface as well as the effects of pore water flow. Therefore, Eq. 4 can be viewed as a conservative estimate of the soil resistance force and an appropriate first approximation for the given application.

In [21] and in the ensuing analysis, a slight seabed indentation is assumed and the depth $d$ is related to the length $x$ of the gouge as $d=x \tan \beta$ yielding the following equation for the soil resistance $\mathrm{P}_{\mathrm{s}}$, i.e.,

$P_{s}=K_{s} x^{2} \tan ^{2} \beta$

where $\beta$ is the seabed slope (assumed to be approximately constant over the space domain of the gouging event). Note also that seabed indentation observations indicate most gouge features are uniform in cross section over long distances on often almost horizontal seabed configurations [29].

Substituting Eq. 3 and Eq. 5 into Eq. 1 yields, 
$m \ddot{x}+\mathrm{K}_{\mathrm{s}} \mathrm{x}^{2} \tan ^{2} \beta=\left(C_{m}-1\right) \rho V_{b} \ddot{x}+\frac{1}{2} C_{d} \rho A_{b}|v-\dot{x}|(v-\dot{x})$

Further, defining the ratios $A=\frac{\frac{1}{2} C_{d} \rho A_{b}}{m_{e}}$ and $B=\frac{K_{s} \tan ^{2} \beta}{m_{e}}$, where

$$
m_{e}=m+\left(C_{m}-1\right) \rho V_{b}
$$

Eq. 6 is rewritten as

$\ddot{\mathrm{x}}+\mathrm{Bx}^{2}=A[v-\dot{x}]^{2}$.

Note that Eq. 8 is actually the equation proposed in [21].

\section{Modified ice gouge model}

Clearly, relying on the physics of the problem, it is anticipated that at time $t=0$ when the berg touches the seabed the conditions $\dot{x}(t=0)=v$ and $x(t=0)=0$ are satisfied. Next, as the gouging process progresses, the velocity $\dot{x}$ of the berg gradually decreases (monotonically) until it becomes zero. Obviously, (and since a constant velocity $v$ of the current is assumed) a reversal of the sign of the berg velocity $\dot{x}$ is not anticipated at any point during the gouging process.

Nevertheless, note that the form of Eq. 8 which can be construed as a nonlinear single degree of freedom oscillator excited by the forcing term $A[v-\dot{x}]^{2}$ suggests a solution of an oscillatory nature. Indeed, for the parameters values shown in Table 1 (the values are within typical ranges as presented in the Discussion section of reference [21]), the model in [21] yields a solution that is plotted in Fig. 1. It can be readily seen that according to Eq. 8 the berg oscillates. Clearly, this is not a physically realistic outcome; and thus, the dynamics modeling described by Eq. 8 needs to be modified. In this 
regard, a large enough energy dissipation term (i.e. damping term) is added to Eq. 8 to prevent any reversal in velocity; this yields

$\ddot{x}+C \dot{x}+B x^{2}=A[v-\dot{x}]^{2}$

where $C$ is the energy dissipation constant. Further, it can be argued that the motion of the berg resembles the non-oscillatory motion of an over-critically damped single degree of freedom oscillator (e.g. [23]). In the following, a simple parametric study is performed to determine the lowest damping coefficient value $C$ which prevents oscillatory motion; thus, being consistent with the physics of the problem. In this regard, in Fig. 1 the solution of Eq. 9 is plotted for several values of the damping coefficient $C$ and compared with the solution of Eq. 8 (model by [21]).

Three energy dissipation constants are considered in the current example for solving Eq.

9. Note that although only three values are considered in the present example, the parametric study requires that an initial value is chosen arbitrarily and then ramped up in order to gauge the amount of damping required to prevent oscillatory behavior. The equations are solved utilizing a standard fourth order Runge-Kutta scheme [30] and the resulting gouge lengths as a function of time are shown in Fig. 1.

As can be seen in Fig. 1 , a constant $C=6 \times 10^{7}$ is required to prevent oscillatory motion. Note that the solution derived using the model by [21] yields the same final gouge length result. However, as previously mentioned, the response oscillates, and thus, does not portray the physics of the problem realistically. Further, due to the oscillatory nature of the response the computational cost related to the ODE solution is 
increased since more time is required for the solution to converge to the final gouge length.

The parametric exercise presented above can be performed for other system parameters as well. Additionally, the above exercise provides some insight regarding an approximate range of the response. This information can be used in the proposed approach to solve the SDE more efficiently (details regarding increasing the efficiency of the proposed approach are presented in the numerical example section of this paper). In the next subsection, the proposed model is extended to consider the uncertainties in soil strength.

\section{Stochastic Model}

Offshore systems most often exhibit random characteristics due to inherent uncertainties in offshore loading conditions (i.e. wind, wave, current, earthquake etc.) and soil properties. Even design standards and codes acknowledge the uncertainty in soil strength (see $[31,32])$. Thus, to realistically capture the system behavior the inherent randomness must be appropriately modeled. This requires a suitable uncertainty quantification methodology with concepts and methods of statistics and probability theory (e.g. [33]). Stochastic procedures have been shown to provide a sound framework for a rational treatment of uncertainties (see [34]). Indicatively, probabilistic approaches have been developed that account for uncertainties in the design of pipeline systems (e.g. [35-38]).

Note, however, that the previous theoretical models developed for estimating gouge depths (i.e. [15-22]) are purely deterministic; thus, neglecting the variations in soil 
properties and utilizing deterministic "mean" (or "extreme" in some cases) values. Consequently, gouge depths may be overestimated when using deterministic approaches, which can lead to costly offshore pipeline burial campaigns. Therefore it is beneficial to model the uncertainty in soil strength as more realistic gouge depths can be estimated, which may result in lower offshore installation costs.

In this regard, (Eq. 9) is modified by considering the randomness in the soil strength and Eq. 9 is re-written as

$\ddot{x}+C \dot{x}+B[1+w(t)] x^{2}=A\left[v_{0}-\dot{x}\right]^{2}$

where $\mathrm{w}(\mathrm{t})$ represents a Gaussian, zero-mean white noise process possessing a power spectrum $S_{0}$. Note that a magnitude for the white noise intensity $S_{0}$ is chosen so as to ensure that the probability of a resulting negative soil resistance is negligible. The modeling of the uncertainty in soil strength as a Gaussian process is recommended in [32]. Also, it is noted that more sophisticated, than the Gaussian white noise, stochastic modeling of the soil strength can be utilized based on available measured data. Potential future work includes modifications of the model to consider uncertainties in other parameters as well such as berg mass, current velocity etc. Clearly, Eq. 10 is a nonlinear SDE with no known exact solution. In the subsequent section the basic elements of the recently developed Wiener Path Integral (WPI) technique is presented which will be used to solve the SDE Eq. 10 in an efficient manner; thus, circumventing computationally demanding MCS. 


\section{WIENER PATH INTEGRAL (WPI) based solution treatment}

The Monte Carlo simulation (e.g. [11]) has been, perhaps, the most versatile tool for determining response and reliability statistics of stochastic systems. However, there are cases, for instance when large-scale complex systems are concerned or when the quantity of interest has a relatively small probability of occurrence, for which the use of MCS techniques can be computationally demanding, or even prohibitive. Thus, there is a need for developing alternative efficient approximate analytical and/or numerical solution techniques; see [39-43] for some recent references.

One of the promising frameworks relates to the concept of the Wiener path integral (WPI). In this regard, note that although the WPI has strongly impacted the field of theoretical physics, the engineering community has so far ignored its potential as a powerful uncertainty quantification tool. The concept of path integral was introduced by [44] and was reinvented in a different form by [45] to reformulate quantum mechanics. A more detailed treatment of path integrals, especially of their applications in physics, can be found in a number of books such as in [46]. Recently, in [24] an approximate analytical WPI technique was developed based on a variational formulation and on the concepts of stochastic averaging/linearization for addressing certain stochastic engineering dynamics problems. In this regard, relying on the concept of the most probable path an approximate expression was derived for the non-stationary response probability density function (PDF). Further, the aforementioned technique was extended in [25] to account for multi-degree-of-freedom (MDOF) systems as well as for 
hysteretic nonlinearities. In [47] the technique was further enhanced and generalized to treat linear and nonlinear systems endowed with fractional derivatives terms.

The basic elements of the WPI technique are presented in more detail below.

The technique is then applied to the ice-gouging problem in the subsequent section.

\section{WPI formulation and Most Probable Path}

Regarding the WPI, it can be realized as a functional integral over the space of all possible paths $C\left\{\mathrm{x}_{\mathrm{i}}, \dot{\mathrm{x}}_{\mathrm{i}}, \mathrm{t}_{\mathrm{i}} ; \mathrm{x}_{\mathrm{f}}, \dot{\mathrm{x}}_{\mathrm{f}}, \mathrm{t}_{\mathrm{f}}\right\}$ starting from a state $\left(\mathrm{x}_{\mathrm{i}}, \dot{\mathrm{x}}_{\mathrm{i}}, \mathrm{t}_{\mathrm{i}}\right)$ and reaching the state $\left(\mathrm{x}_{\mathrm{f}}, \dot{\mathrm{x}}_{\mathrm{f}}, \mathrm{t}_{\mathrm{f}}\right)$, where $\mathrm{x}\left(\mathrm{t}_{\mathrm{i}}\right)=\mathrm{x}_{\mathrm{i}}, \dot{\mathrm{x}}\left(\mathrm{t}_{\mathrm{i}}\right)=\dot{\mathrm{x}}_{\mathrm{i}}, \mathrm{x}\left(\mathrm{t}_{\mathrm{f}}\right)=\mathrm{x}_{\mathrm{f}}, \dot{\mathrm{x}}\left(\mathrm{t}_{\mathrm{f}}\right)=\dot{\mathrm{x}}_{\mathrm{f}}$. It possesses a probability distribution on the path space as its integrand, which is denoted by $\mathrm{W}[\mathrm{x}(\mathrm{t})]$ and is called probability density functional. In this manner, the transition PDF $\mathrm{p}\left(\mathrm{x}_{\mathrm{f}}, \dot{\mathrm{x}}_{\mathrm{f}}, \mathrm{t}_{\mathrm{f}} \mid \mathrm{x}_{\mathrm{i}}, \dot{\mathrm{x}}_{\mathrm{i}}, \mathrm{t}_{\mathrm{i}}\right)$ is given by

$p\left(x_{f}, \dot{x}_{f}, t_{f} \mid x_{i}, \dot{x}_{i}, t_{i}\right)=\int_{\left\{x_{i}, \dot{x}_{i}, t_{i}\right\}}^{\left\{x_{i}, \dot{x}_{i}, t_{f}\right\}} W[x(t)][d x(t)]$.

Further, note that even if the probability density functional is constructed, the analytical solution of the WPI of Eq. 11 is a rather challenging task. Thus, to circumvent the aforementioned challenge, several research efforts have focused on developing approximate techniques for determining the transition PDF $p\left(x_{f}, \dot{\mathrm{x}}_{\mathrm{f}}, \mathrm{t}_{\mathrm{f}} \mid \mathrm{x}_{\mathrm{i}}, \dot{\mathrm{x}}_{\mathrm{i}}, \mathrm{t}_{\mathrm{i}}\right)$. In this regard, researchers invoked a variational formulation and defined a Lagrangian function $\mathrm{L}(\mathrm{x}, \dot{\mathrm{x}}, \ddot{\mathrm{x}})$ for determining the most probable path that connects the points $\left(\mathrm{x}_{\mathrm{i}}, \dot{\mathrm{x}}_{\mathrm{i}}, \mathrm{t}_{\mathrm{i}}\right)$ and $\left(\mathrm{x}_{\mathrm{f}}, \dot{\mathrm{x}}_{\mathrm{f}}, \mathrm{t}_{\mathrm{f}}\right)$. In this manner, a variational principle can lead to the associated EulerLagrange equation to be solved for the most probable path; see $[24,25]$ for a more detailed presentation. 
Specifically, utilizing the Lagrangian function and considering Eq. 11 yields (e.g. [46])

$\mathrm{W}[\mathrm{x}(\mathrm{t})]=\operatorname{Dexp}\left(-\int_{\mathrm{t}_{\mathrm{i}}}^{\mathrm{t}_{\mathrm{f}}} \mathrm{L}(\mathrm{x}, \dot{\mathrm{x}}, \ddot{\mathrm{x}}) \mathrm{dt}\right)$

where $\mathrm{D}$ is a normalization coefficient. The largest contribution to the Wiener path integral comes from the trajectory for which the integral in the exponential (Eq. 12) becomes as small as possible. Variational calculus rules (e.g. [48]) dictate that this trajectory with fixed end points satisfies the extremality condition

$\delta \int_{t_{i}}^{t_{f}} L\left(x_{c}, \dot{x_{c}}, \ddot{x}_{c}\right) d t=0$.

This condition leads to the E-L equation

$\frac{\partial \mathrm{L}}{\partial \mathrm{x}_{\mathrm{c}}}-\frac{\partial}{\partial \mathrm{t}} \frac{\partial \mathrm{L}}{\partial \dot{\mathrm{x}}_{\mathrm{c}}}+\frac{\partial^{2}}{\partial \mathrm{t}^{2}} \frac{\partial \mathrm{L}}{\partial \ddot{\mathrm{x}}_{\mathrm{c}}}=0$,

with the four boundary conditions

$\mathrm{x}_{\mathrm{c}}\left(\mathrm{t}_{\mathrm{i}}\right)=\mathrm{x}_{\mathrm{i}}, \dot{\mathrm{x}}_{\mathrm{c}}\left(\mathrm{t}_{\mathrm{i}}\right)=\dot{\mathrm{x}}_{\mathrm{i}}, \mathrm{x}_{\mathrm{c}}\left(\mathrm{t}_{\mathrm{f}}\right)=\mathrm{x}_{\mathrm{f}}, \dot{\mathrm{x}}_{\mathrm{c}}\left(\mathrm{t}_{\mathrm{f}}\right)=\dot{\mathrm{x}}_{\mathrm{f}}$,

where $\mathrm{x}_{\mathrm{c}}$ represents the most probable trajectory. Next, solving the boundary value problem (BVP) of Eq. 14 together with the conditions of Eq. 15 (e.g. [49]) yields a solution for the transition PDF $p\left(x_{f}, \dot{x}_{f}, t_{f} \mid x_{i}, \dot{x}_{i}, t_{i}\right)$ in the form

$\mathrm{p}\left(\mathrm{x}_{\mathrm{f}}, \dot{\mathrm{x}}_{\mathrm{f}}, \mathrm{t}_{\mathrm{f}} \mid \mathrm{x}_{\mathrm{i}}, \dot{\mathrm{x}}_{\mathrm{i}}, \mathrm{t}_{\mathrm{i}}\right)=\mathrm{D} \exp \left[-\int_{\mathrm{t}_{\mathrm{i}}}^{\mathrm{t}_{\mathrm{f}}} \mathrm{L}\left(\mathrm{x}_{\mathrm{c}}, \dot{\mathrm{x}}_{\mathrm{c}}, \ddot{\mathrm{x}}_{\mathrm{c}}\right) \mathrm{dt}\right]$

It can be readily seen that for fixed time points $t_{i}$ and $t_{f}$, $D$ can be determined by merely applying the normalization condition

$\int_{-\infty}^{\infty} \int_{-\infty}^{\infty} p\left(x_{f}, \dot{x}_{f}, t_{f} \mid x_{i}, \dot{x}_{i}, t_{i}\right) d x_{f} d \dot{x}_{f}=1$

The primary approximation of the technique relates to the fact that only the most probable path $\mathrm{x}_{\mathrm{c}}$ is considered in the evaluation of the functional integral of Eq. 10 instead of all the possible paths $C\left\{\mathrm{x}_{\mathrm{i}}, \dot{\mathrm{x}}_{\mathrm{i}}, \mathrm{t}_{\mathrm{i}} ; \mathrm{x}_{\mathrm{f}}, \dot{\mathrm{x}}_{\mathrm{f}}, \mathrm{t}_{\mathrm{f}}\right\}$. The concept of the most probable 
path can be viewed as something equivalent to the fact that the most probable value of a random variable is the one corresponding to the peak of the PDF.

\section{WPI Numerical implementation elements}

It is worth noting that for linear systems, the boundary value problem of Eq. 14 and Eq.

15 can be solved analytically, yielding an explicit closed-form expression for the most

probable path $\mathrm{x}_{\mathrm{c}}$. Unfortunately, for the case of nonlinear systems (e.g. the current ice gouging problem), the BVP of Eq. 14 and Eq. 15 cannot, in general, be solved

analytically; thus, a numerical solution technique needs to be implemented. In this regard, for a given time instant $t_{f}$ and a given vector value $\left(\mathrm{x}_{\mathrm{f}}, \dot{\mathrm{x}}_{\mathrm{f}}\right)$, a numerical solution of Eq. 14 and Eq. 15 yields a single point of the response PDF via Eq. 16. Typically, an effective domain of values is assumed for the response PDF $p\left(x_{f}, \dot{x}_{f}, t_{f} \mid x_{i}, \dot{x}_{i}, t_{i}\right)$; namely, for the ith components $\mathrm{x}_{\mathrm{i}, \mathrm{f}}$ of $\mathrm{x}_{\mathrm{f}}$ and $\dot{\mathrm{x}}_{\mathrm{i}, \mathrm{f}}$ of $\dot{\mathrm{x}}_{\mathrm{f}}$ it is assumed that $\mathrm{x}_{\mathrm{i}, \mathrm{f}}=\in\left[\mathrm{x}_{\mathrm{i}, \mathrm{f}, \mathrm{min}}, \mathrm{x}_{\mathrm{i}, \mathrm{f}, \mathrm{max}}\right]$, $\mathrm{x}_{\mathrm{i}, \mathrm{f}}^{\mathrm{j}}=\mathrm{x}_{\mathrm{i}, \mathrm{f} \text {,min }}+(\mathrm{j}-1) \Delta \mathrm{x}_{\mathrm{i}, \mathrm{f}}, \mathrm{j}=1, \ldots, \mathrm{n}$ with $\Delta \mathrm{x}_{\mathrm{i}, \mathrm{f}}=\left(\mathrm{x}_{\mathrm{i}, \mathrm{f}, \mathrm{max}}-\mathrm{x}_{\mathrm{i}, \mathrm{f}, \mathrm{min}}\right) /(\mathrm{n}-1)$ and $\dot{\mathrm{x}}_{\mathrm{i}, \mathrm{f}}=\in\left[\dot{\mathrm{x}}_{\mathrm{i}, \mathrm{f}, \min }, \dot{\mathrm{x}}_{\mathrm{i}, \mathrm{f}, \mathrm{max}}\right], \dot{\mathrm{x}}_{\mathrm{i}, \mathrm{f}}=\dot{\mathrm{x}}_{\mathrm{i}, \mathrm{f} \text { min }}+(\mathrm{j}-1) \Delta \dot{\mathrm{x}}_{\mathrm{i}, \mathrm{f}}, \mathrm{j}=1, \ldots, \mathrm{n}$ with $\Delta \dot{\mathrm{x}}_{\mathrm{i}, \mathrm{f}}=\left(\dot{\mathrm{x}}_{\mathrm{i}, \mathrm{f}, \max }-\right.$ $\left.\dot{\mathrm{x}}_{\mathrm{i}, \mathrm{f}, \mathrm{min}}\right) /(\mathrm{n}-1)$, respectively. It can be readily seen that in the general case, where no analytical solution exists for Eq. 14 and Eq. 15, the determination of the system response PDF can be computationally demanding. Specifically, the number of BVPs of the form of Eq. 14 and Eq. 15 to be solved is $n^{2}$. However, for the ice-gouging problem, only $n$ BVPs are required to compute the response PDF of maximum gouge depth due to the fact that the final velocity is known (i.e. $\dot{x}_{c}\left(t_{f}\right)=0$ ). Note that based on numerical examples performed in this study, a value of $n=50$ has been deemed more than 
adequate for determining the system response PDF with reasonable accuracy in most cases.

\section{STOCHASTIC ICE GOUGING PROBLEM}

The WPI approach presented in the previous section is now applied to the icegouging problem. Solving Eq. 10 for $w(t)$ yields,

$$
w(t)=\frac{A v_{0}^{2}-B x^{2}-\frac{c}{m} \dot{x}-2 A v_{0} \dot{x}+A \dot{x}^{2}-\ddot{x}}{B x^{2}}
$$

The probability density functional for the white noise process $w(t)$ is given by (e.g. [46])

$\mathrm{W}[\mathrm{w}(\mathrm{t})]=\operatorname{Dexp}\left(-\int_{\mathrm{t}_{\mathrm{i}}}^{\mathrm{t}_{\mathrm{f}}} \frac{1}{2} \frac{\mathrm{w}(\mathrm{t})^{2}}{2 \pi \mathrm{S}_{0}} \mathrm{dt}\right)$

Next, Eq. 18 is substituted into Eq. 19 and the probability density functional $W[w(t)]$ is interpreted as the probability density functional $\mathrm{W}[\mathrm{x}(\mathrm{t})]$ for $\mathrm{x}(\mathrm{t})$, yielding

$W[w(t)]=\operatorname{Dexp}\left(-\int_{t_{i}}^{t_{f}} \frac{1}{4 \pi S_{0}}\left(\frac{A_{v_{0}}^{2}-B x^{2}-\frac{c}{m} \dot{x}-2 A v_{0} \dot{x}+A \dot{x}^{2}-\ddot{x}}{B x^{2}}\right)^{2} d t\right)$

Thus, the corresponding Lagrangian is given by

$L(X, \dot{X}, \ddot{X})=\frac{1}{4 \pi S_{0}}\left(\frac{A_{0}{ }^{2}-B x^{2}-\frac{c}{m} \dot{x}-2 A v_{0} \dot{x}+A \dot{x}^{2}-\ddot{x}}{B x^{2}}\right)^{2}$.

Substituting Eq. 21 into Eq. 14 and considering Eq. 15 leads to the E-L equation,

$$
\begin{aligned}
& \frac{1}{2 B^{2} m^{2} \pi S_{0} x_{c}^{6}}\left(-20 m \dot{x}_{c}^{2}\left(-\left(c+2 A m v_{0}\right) \dot{x}_{c}+A m \dot{x}_{c}^{2}+m\left(A v_{0}^{2}-\ddot{x}_{c}\right)\right)+2 B m^{2} x_{c}^{3}\left(A v_{0}^{2}-A \dot{x}_{c}^{2}-\right.\right. \\
& \left.2 \ddot{x}_{c}\right)+2 A B m^{2} x_{c}^{4} \ddot{x}_{c}-2 x_{c}\left(4 A m\left(c+2 A m v_{0}\right) \dot{x}_{c}^{3}-3 A^{2} m^{2} \dot{x}_{c}^{4}-\dot{x}_{c}^{2}\left(c^{2}+4 c m v_{0}+6 A^{2} m^{2} v_{0}^{2}+\right.\right. \\
& \left.\left.8 A m^{2} \ddot{x}_{c}\right)+m^{2}\left(A^{2} v_{0}^{4}-4 A v_{0}^{2} \ddot{x}_{c}+3 \ddot{x}_{c}^{2}\right)+2 m \dot{x}_{c}\left(3\left(c+2 A m v_{0}\right) \ddot{x}_{c}+2 m x_{c}^{(3)}\right)\right)+x_{c}^{2}\left(-\left(c^{2}+\right.\right. \\
& \left.\left.\left.4 A c m v_{0}+6 A^{2} m^{2} v_{0}^{2}\right) \ddot{x}_{c}+6 A m\left(c+2 A m v_{0}\right) \dot{x}_{c} \ddot{x}_{c}+6 m^{2} \dot{x}_{c}^{2}\left(B-A^{2} \ddot{x}_{c}\right)+m^{2} x_{c}^{(4)}\right)\right)=0
\end{aligned}
$$

where $x_{c}^{(3)}$ and $x_{c}^{(4)}$ denote the third and fourth derivative with time. Further, the boundary conditions are $\mathrm{x}_{\mathrm{c}}(0)=0$ and $\dot{\mathrm{x}}_{\mathrm{c}}(0)=\mathrm{v}_{\mathrm{c}}$. The most probable path, $\mathrm{x}_{\mathrm{c}}$, is determined by solving the BVP of Eq. 22 numerically (e.g. [49]). 


\section{Numerical example}

A numerical example is presented to demonstrate the efficiency of the developed approach. The system parameters listed in Table 1 along with a damping coefficient of $6.0 \times 10^{\wedge} 7$ (derived previously and shown in Fig. 1) are used for the current example. The zero mean white noise process (modeling the deviation of the soil strength from its mean value) is assumed to have a power spectrum value of $S_{0}=0.01$, which is chosen so that the probability of a resulting negative soil resistance is negligible. Further, the parametric exercise used to compute the energy dissipation term provides some insight into the effective domain of the response PDF (i.e. approximate maximum and minimum gouge length/depth). This improves the efficiency of the approach as a relatively smaller amount of BVPs need to be solved to achieve the desired discretization density of the PDF. Consequently, an approximate range of values of 202 meters to 206 meters (i.e. $x_{i, f, m i n}$ and $x_{i, f, \max }$ ) for the gouge length response PDF is chosen and the numerical technique presented in subsection "WPI Numerical implementation elements" is employed. Next, a value of $n=50$ BVPs are solved and the resulting length response PDF is shown in Fig. 2. Since the gouge length PDF is computed, a transformation is conducted (e.g. [33]) to convert it to a response PDF for gouge depth (according to the relationship $d=x \tan \beta$ ) assuming a seabed slope of $1 / 100$, a value within a typical range as presented in [21].

To validate the approach, a Monte Carlo simulation is conducted and Eq. 9 is solved using a standard fourth order Runge-Kutta integration scheme (e.g. [30]) and utilizing 50,000 realizations. Fig. 3 shows the gouge depth response PDFs derived using the WPI 
and MCS approaches. As can be seen in the figure, a satisfactory level of accuracy is achieved using the WPI technique.

The approach can also be used to derive the response PDFs at different time instances if needed. Fig. 4 shows the gouge depth response PDF as a function of time derived using the MCS and Fig. 5 shows the comparison of the response PDFs derived using WPI and MCS approaches at various time instances. As can be seen in the figures, a satisfactory level of accuracy is achieved with the WPI approach. It is worth noting that for Fig. 5 an approximate range of values for the final velocity were required (i.e. $\dot{\mathrm{x}}_{\mathrm{i}, \mathrm{f}, \mathrm{min}}$, and $\dot{\mathrm{x}}_{\mathrm{i}, \mathrm{f}, \mathrm{max}}$ ) to solve $\mathrm{n}^{\wedge} 2$ BVPs and to compute the response PDFs at the different time instances. This, of course, is due to the fact that the final velocity is unknown at the intermediate time instances.

\section{Discussion}

The maximum gouge depth response PDF is computed by solving $n=50$ boundary value problems only. Obviously, the computational cost of the developed technique increases with an increasing value of $n$, or, in other words, when a relatively denser discretization map is utilized. Nevertheless, it is noted that to obtain reliable response PDF estimates via MCS, especially in the tails of the PDF where samples occur with low probability, the number of realizations to be produced and of subsequent numerical integrations of Eq. 9 needs to be large (on the order of $10^{6}$ simulations). To provide an order of magnitude for the example presented above, the 50 BVPs that were solved for the WPI approach required only a few minutes (i.e. between 1 to 2 minutes) of computational time while 
the MCS approach required several hours (i.e. between 6 to 7 hours) of computational time using the same machine.

Additionally, note that the proposed approach is befitting for determining the maximum gouge depth as only $\mathrm{n}$ BVPs are required versus $\mathrm{n}^{2}$ (which would be the case in general stochastic response determination problems, e.g. [24]). This is due to the fact that the final velocity is zero.

The current model can be modified to consider the uncertainty in other parameters as well such as berg mass, current velocity etc. Also, it is envisioned that future work will consider more sophisticated stochastic modeling as well as system modeling.

\section{CONCLUDING REMARKS}

Ice gouging has been identified as a concern for oil and gas development in the arctic and thus has prompted efforts to develop mathematical models of the gouging event (e.g. [22]). Accurate theoretical models are required to circumvent the costly seabed survey campaigns. In the present paper, a previous formulation governing the ice gouging has been enhanced and then extended to consider the uncertainties in the soil strength. Further, the recently developed Wiener path integral technique for treating certain random vibration problems has been applied to solve the SDE governing the ice gouging motion. Specifically, a Lagrangian formulation in conjunction with a variational principle has been utilized to derive an Euler-Lagrange equation governing the most probable response trajectory. The boundary value problem has then been solved numerically and the corresponding response probability density function has 
been derived. The proposed approach is especially well suited for determining the maximum gouge depth as only $\mathrm{n}$ boundary value problems need to be solved as opposed to $\mathrm{n}^{2}$ (which would be the case in general stochastic response determination problems, e.g. [24]), due to the fact that the final velocity is known (i.e. $\dot{x}_{c}\left(t_{f}\right)=0$ ).

Further, the reliability of the approach has been demonstrated by comparing the results to those obtained by Monte Carlo simulations. The resulting PDFs computed via the WPI compare well to those computed using the more computationally demanding MCS-based approach.

The advantage of the proposed stochastic dynamics based approach over other approaches is computational efficiency, which hinges on the simplified model adopted to estimate the gouge depth coupled with the efficient WPI based solution used to conduct the stochastic analysis. The accuracy of sophisticated FEM based approaches is not necessarily higher than that of more approximate analytical approaches since a high level of uncertainty is involved in the selection of parameter values related to the system and to the excitation/environment. Further, parameter uncertainty is more prevalent in the beginning stages of projects when in-situ data is limited or not available. As such, it is envisaged that the developed approach can be used during the "desk-top" study or "front-end" phases of projects given the high level of uncertainty involved in the selection of parameter values related to the system and to the excitation/environment in these phases. During the later stages of projects (i.e. detailed design, execution etc.) when in-situ data is gathered and the level of uncertainty is decreased, the use of more computationally demanding FEM based models is 
recommended. Nonetheless, the developed approach can provide a good first approximation of the gouge length/depth. Further, parametric studies are feasible using the developed approach since it is orders of magnitude more efficient than the common MCS-based approaches currently used in the industry for system response and reliability analysis.

Finally, note that although path integrals have reformulated and revolutionized theoretical physics, the engineering mechanics community has neglected their potential for uncertainty quantification of systems of engineering interest. It is hoped that the WPI will offer a potent new tool for treating complex offshore oil and gas problems where uncertainties are prevalent. 


\section{REFERENCES}

[1] United States Geological Survey (USGS), 2008, "90 Billion Barrels of Oil and 1,670 Trillion Cubic Feet of Natural Gas Assessed in the Arctic."

[2] OG21 Strategy Report, 2006, "Technology Strategy for the Arctic," Technical Report, Oil and Gas in the 21 Century, The Research Council of Norway.

[3] Barrette, P., 2011, “Offshore Pipeline Protection Against Seabed Gouging By Ice: An Overview," Cold Regions Science and Technology, 69(1), pp. 3-20.

[4] Abdalla, B., Jukes, P., Eltaher, A., and Duron, B., 2008, "The Technical Challenges of Designing Oil and Gas Pipelines in the Arctic," Oceans '08 MTS/IEEE, Quebec City, QC.

[5] Abdalla, B., Pike, K., Eltaher, A., Jukes, J. and Duron, B., 2009, "Development And Validation Of A Coupled Eulerian Lagrangian Finite Element Ice Scour Model," Proceedings $28^{\text {th }}$ International Conference on Ocean, Offshore and Arctic Engineering, 5, pp. 87-95.

[6] Banneyake, R., Hossain, M. K., Eltaher, A., Nguyen, T., \& Jukes, P., 2011, "Ice-SoilPipeline Interactions Using Coupled Eulerian-Lagrangian (CEL) Ice Gouge Simulations Extracts From Ice Pipe JIP," Offshore Technology Conference, OTC-22047-MS.

[7] Jukes, P., Eltaher, A., Abdalla, B. and Duron, B., 2008, "The Design and Simulation of Arctic Subsea Pipelines - Ice Gouging Formulations," 4th Annual Arctic Oil \& Gas Conference, Oslo, Norway.

[8] Konuk, I.S. and Gracie, R., 2004, "A 3-Dimensional Eulerian FE Model for Ice Scour," International Pipeline Conference, Calgary, Alberta, IPC2004-0075, pp. 1911-1918.

[9] Konuk, I.S., Yu, S. Gracie, R., 2005, “An ALE FEM Model of Ice Scour," Proc. 11th International Conference of the International Association of Computer Methods and Advance in Geomechanics, Turin, Italy, 3, pp. 63-70.

[10] Phillips, R., Barrett, J. and Al-Showaiter, A., 2010, "Ice Keel-Seabed Interaction: Numerical Modeling Validation," Offshore Technology Conference, OTC2010-20696.

[11] Liferov, P., Shkhinek, K. N., Vitali, L., \& Serre, N., 2007, "Ice Gouging Study - Actions And Effects," Port and Ocean Engineering Under Arctic Conditions POAC-07, Dalian, China, ISBN 978-7-5611-3631-7, pp. 774-786.

[12] Rubinstein R. Y., Kroese D. P., 2007, Simulation and the Monte Carlo Method, John Wiley \& Sons, Hoboken.

[13] Au S.K., Wang Y., 2014, Engineering Risk Assessment with Subset Simulation, J. Wiley \& Sons, Singapore, Chap. 2. 
[14] Palmer, A.C., Niedoroda, A., 2005, "Ice Gouging and Pipelines: Unresolved Questions," Proceedings of the 18th Conference on Ports and Ocean Engineering Under Arctic Conditions (POAC), Potsdam, NY, USA, 1, pp. 11-22.

[15] Chari, T.R., 1975, "Some Geotechnical Aspects of Iceberg Grounding," Ph.D. Thesis, Memorial University of Newfoundland, St. John's, Canada.

[16] Chari, T.R., 1979, "Geotechnical Aspects of Iceberg Scours on Ocean Floors," Canadian Geotechnical Journal, 16(2), pp. 379-390.

[17] Chari, T.R., Peters, G.R., Muthukrishnaiah, K., 1980, "Hydrodynamic Effects on Iceberg Gouging," Cold Regions Science and Technology, 1(3-4): pp. 223-230.

[18] Been, K., Kosar, K., Hachey, J., Rogers, B.T. \& Palmer, A.C., 1990, "Ice Scour Models," International Conference on Offshore Mechanics and Arctic Engineering, 5, pp. 179 - 188.

[19] Croasdale, K., Comfort, G. \& Been. K., 2005, "Investigation of Ice Limits to Ice Gouging," Proceedings. 18th International Conference on Port and Ocean Engineering Under Arctic Conditions, 1, pp. 23 - 32.

[20] Kioka, S., Saeki, H., 1995, "Mechanics of Ice Gouging." Proceedings 5th International Offshore and Polar Engineering Conference (ISOPE), 2, pp. 398-402.

[21] Lopez, R., Chari, T.R., Moore, E., Peters, G.R., Zielinski, A., 1981, "Environmental Factors Affecting Iceberg Scour Estimates," Cold Regions Science and Technology, 4(1), pp. 55-61.

[22] Walter, D. J., Phillips, R., 1998, "PRISE - Force models for Drained and Undrained Steady State Ice Scouring," Technical Report, C-CORE Publications 98-C33.

[23] Chopra, A. K., 1995, Dynamics of Structures Theory and Applications to Earthquake Engineering, Prentice Hall, Upper Saddle River, NJ. Chap. 3.

[24] Kougioumtzoglou I. A., Spanos P. D., 2012, "An Analytical Wiener Path Integral Technique for Non-Stationary Response Determination of Nonlinear Oscillators," Probabilistic Engineering Mechanics, 28, pp. 125-131.

[25] Kougioumtzoglou, I. A., Spanos P. D., 2014, "Nonstationary Stochastic Response Determination of Nonlinear Systems: A Wiener Path Integral Formalism," J. Eng. Mech., 10.1061/(ASCE)EM.1943-7889.0000780, 04014064.

[26] Morison, J.R., Johnson, J.W., Shaaf, S.A., 1950, "The Force Exerted by Surface Waves on Piles," Journal of Petroleum Technology, 2, pp. 149-154.

[27] Terzaghi, K., Peck, R. B., and Mesri, G., 1996, Soil Mechanics in Engineering Practice, $3^{\text {rd }}$ Edition, Wiley, New York, Chap. 8. 
[28] Coyne, J.C., Lewis, G.W., 1999, "Analysis of Plowing Forces for a Finite-Width Blade in Dense, Ocean Bottom Sand," OCEANS '99 MTS/IEEE, 1, pp. 1-10.

[29] Palmer, A., Konuk, I., Love, J., Been, K. and Comfort, G., 1989, "Ice Scour Mechanics," A Research Paper prepared for Canada Oil and Gas Lands Administration and Gulf Canada Resources Ltd.

[30] Shampine, L. F., 1994, Numerical Solution of Ordinary Differential Equations, Chapman \& Hall, New York, Chap. 4.

[31] Det Norske Veritas, 2010, Environmental Conditions and Environmental Loads, DNVRP-C205.

[32] Det Norske Veritas, 2012, Statistical Representation of Soil Data, DNV-RP-C207.

[33] Ang, A.S., Tang, W.H., 2007, Probability Concepts in Engineering, J. Wiley \& Sons, NJ, Chap. 1 and Chap. 4.

[34] Schuëller, G.I., 2007, "On the Treatment of Uncertainties in Structural Mechanics and Analysis," Computers and Structures, 85, pp. 235-243.

[35] King, T., Phillips, R., Barrett, J., Sonnichsen, G., 2009, "Probabilistic Pipeline Burial Analysis for Protection Against Ice Scour," Cold Regions Science and Technology 59(1), pp. 58-64.

[36] Kenny, S., Barrett, J., Phillips, R. and Popescu, R., 2007, “Integrating Geohazard Demand and Structural Capacity Modeling within a Probabilistic Design Framework for Offshore Arctic Pipelines," Proceedings Seventeenth International Ocean and Polar Engineering Conference (ISOPE), Lisbon, Portugal, ISOPE2007-SBD-03.

[37] Sicilia, C., Bonnet, E., \& Cooper, P. A., 2014, "Probabilistic Lateral Buckling Assessment," Proceedings Twenty-fourth International Ocean and Polar Engineering Conference (ISOPE), Busan, Korea, 25, pp. 241-246.

[38] Gomes, W., T. Beck, A., 2014, "Optimal Inspection and Design of Onshore Pipelines Under External Corrosion Process," Structural Safety, 47, pp. 48-58.

[39] Li J., Chen j., 2009, Stochastic Dynamics of Structures, J. Wiley \& Sons, Singapore, Chap. 5 and Chap 6.

[40] Kougioumtzoglou I. A., 2013, "Stochastic Joint Time-Frequency Response Analysis of Nonlinear Structural Systems," Journal of Sound and Vibration, 332, pp. 7153-7173.

[41] Spanos P. D., Kougioumtzoglou I. A., 2014, "Survival Probability Determination of Nonlinear Oscillators Subject to Evolutionary Stochastic Excitation," Journal of Applied Mechanics, 81, pp. 1-9. 
[42] Kougioumtzoglou I. A., Spanos P. D., 2009, "An Approximate Approach for Nonlinear System Response Determination Under Evolutionary Stochastic Excitation," Current Science, 97, pp. 1203-1211.

[43] Kougioumtzoglou I. A., Spanos P. D., 2013, "Nonlinear MDOF System Stochastic Response Determination via a Dimension Reduction Approach," Computers and Structures, 126, pp. 135-148.

[44] Wiener N., 1921, "The Average of an Analytic Functional," Proc. Natl. Acad. Sci. USA 7(9): 253-260.

[45] Feynman R. P., 1948, "Space-Time Approach to Non-Relativistic Quantum Mechanics," R. M. Phys., 20, pp. 367-387.

[46] Chaichian M., Demichev A., 2001, Path integrals in Physics, Vol. I, Stochastic Processes and Quantum Mechanics, Institute of Physics Publishing, Bristol and Philadelphia.

[47] Di Matteo A., Kougioumtzoglou I. A., Pirrotta A., Spanos P. D., Di Paola M., 2014, "Non-Stationary Stochastic Response Determination Of Nonlinear Oscillators with Fractional Derivatives Elements via the Wiener Path Integral," Probabilistic Engineering Mechanics, 38, pp. 127-135.

[48] Ewing G. M., 1985, Calculus of Variations with Applications, Dover, New York, Chap. 4.

[49] De Coster C., Habets P., 2006, Two-Point Boundary Value Problems: Lower and Upper Solutions, Elsevier, Amsterdam, Chap. 1. 


\section{Figure Captions List}

Fig. 1

Fig. 2

Fig. 3

Fig. 4

Fig. 5
Model by 0 vs. Proposed Model

$$
\text { Gouge Length Response PDF (WPI Approach) }
$$

Maximum Gouge Depth Response PDF (WPI Approach vs. MCS)

Gouge Depth Response PDF with Time (MCS)

Gouge Depth Response PDF at Different Time Instances (WPI Approach

vs. MCS) 


\section{Table Caption List}

\section{Table 1 Inputs used in Numerical Example}




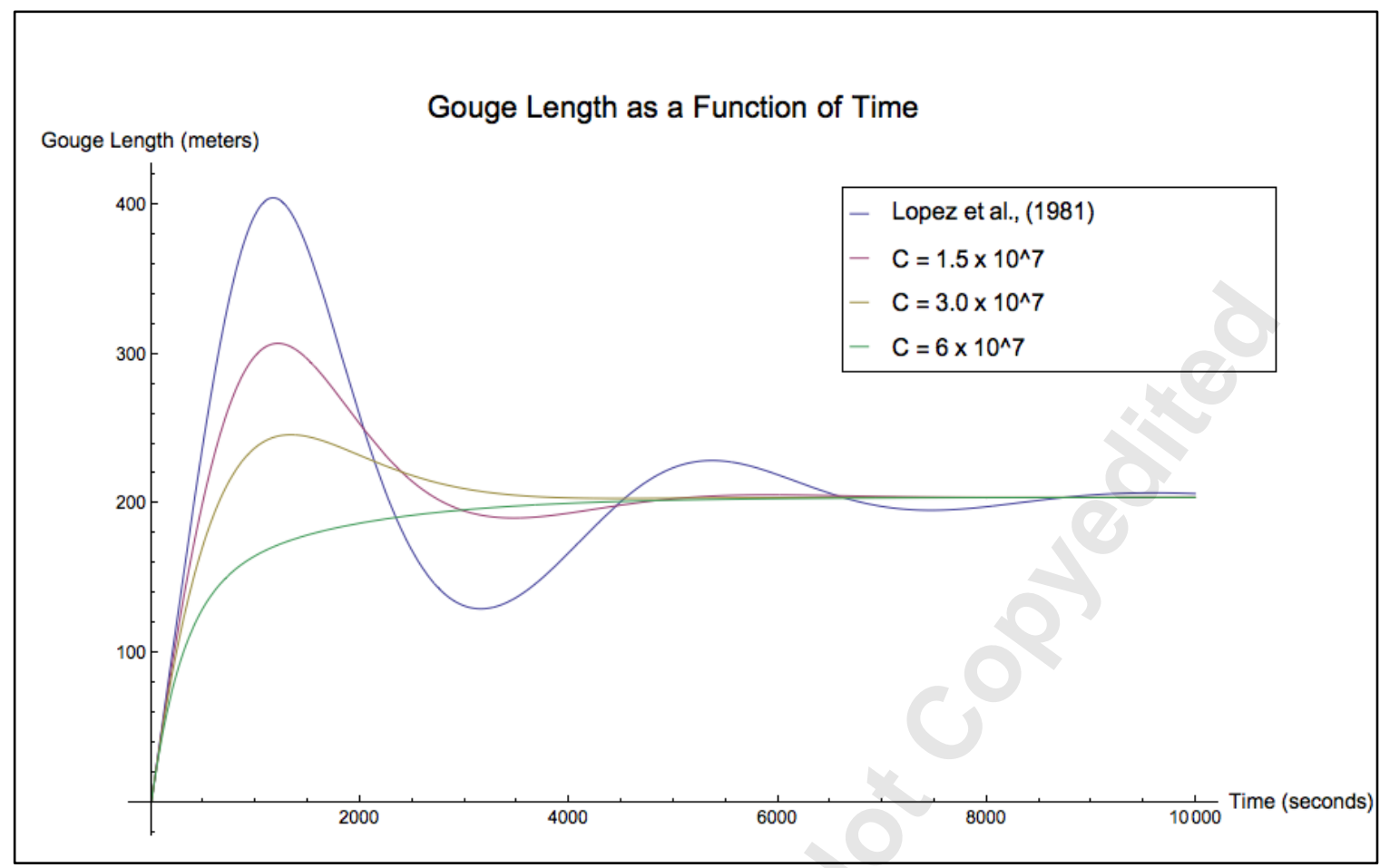

Fig.1. Model by 0 vs. Proposed Model 


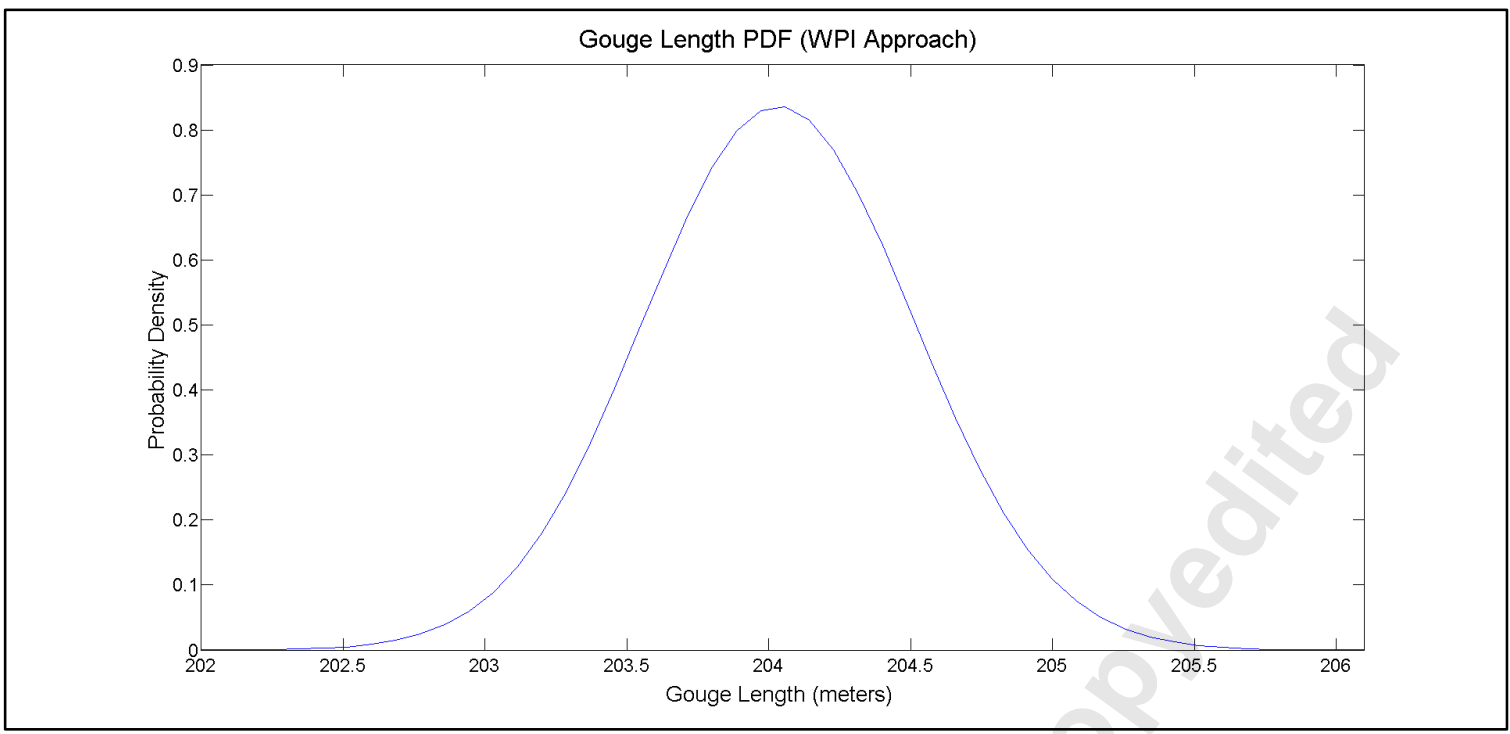

Fig.2. Gouge Length Response PDF (WPI approach) 


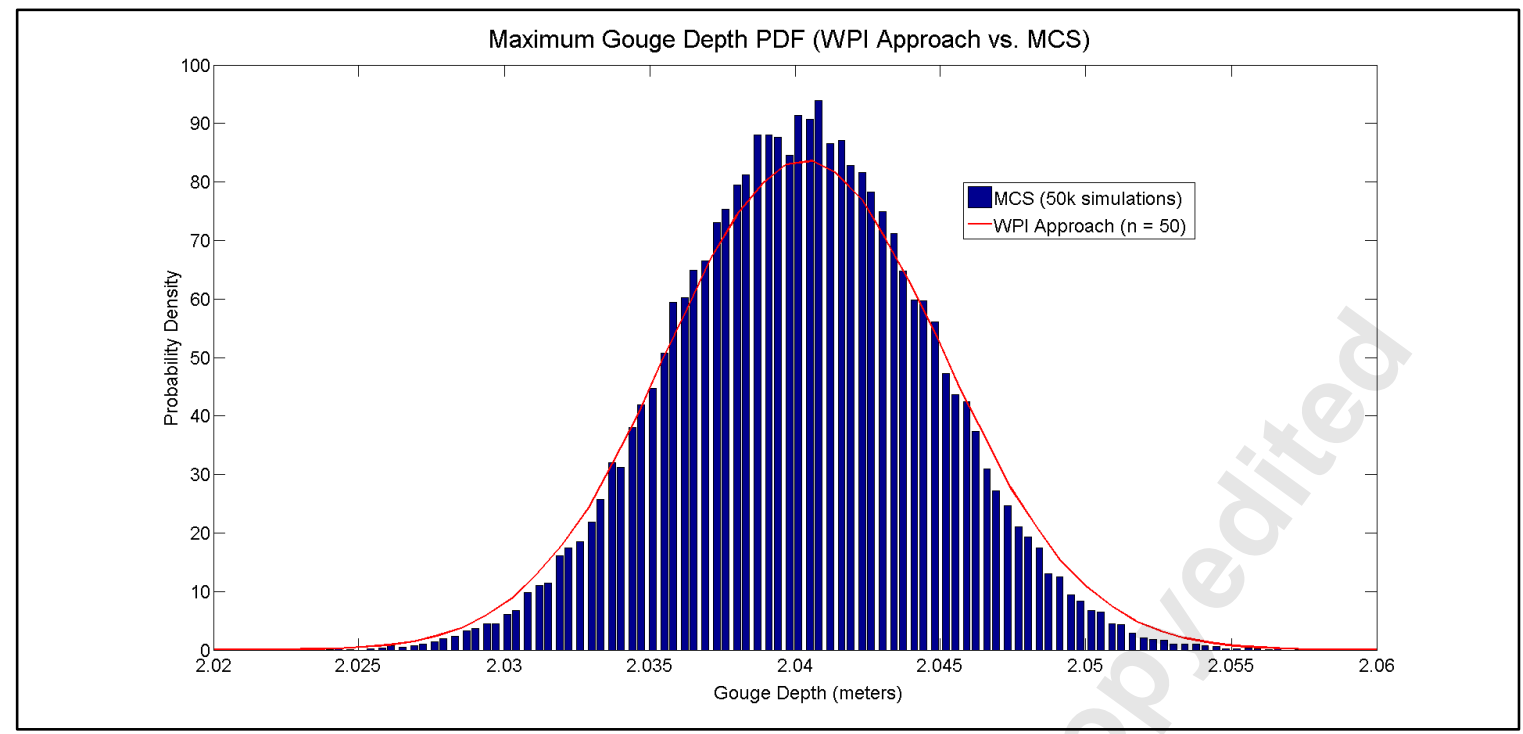

Fig.3. Maximum Gouge Depth Response PDF (WPI Approach vs. MCS) 


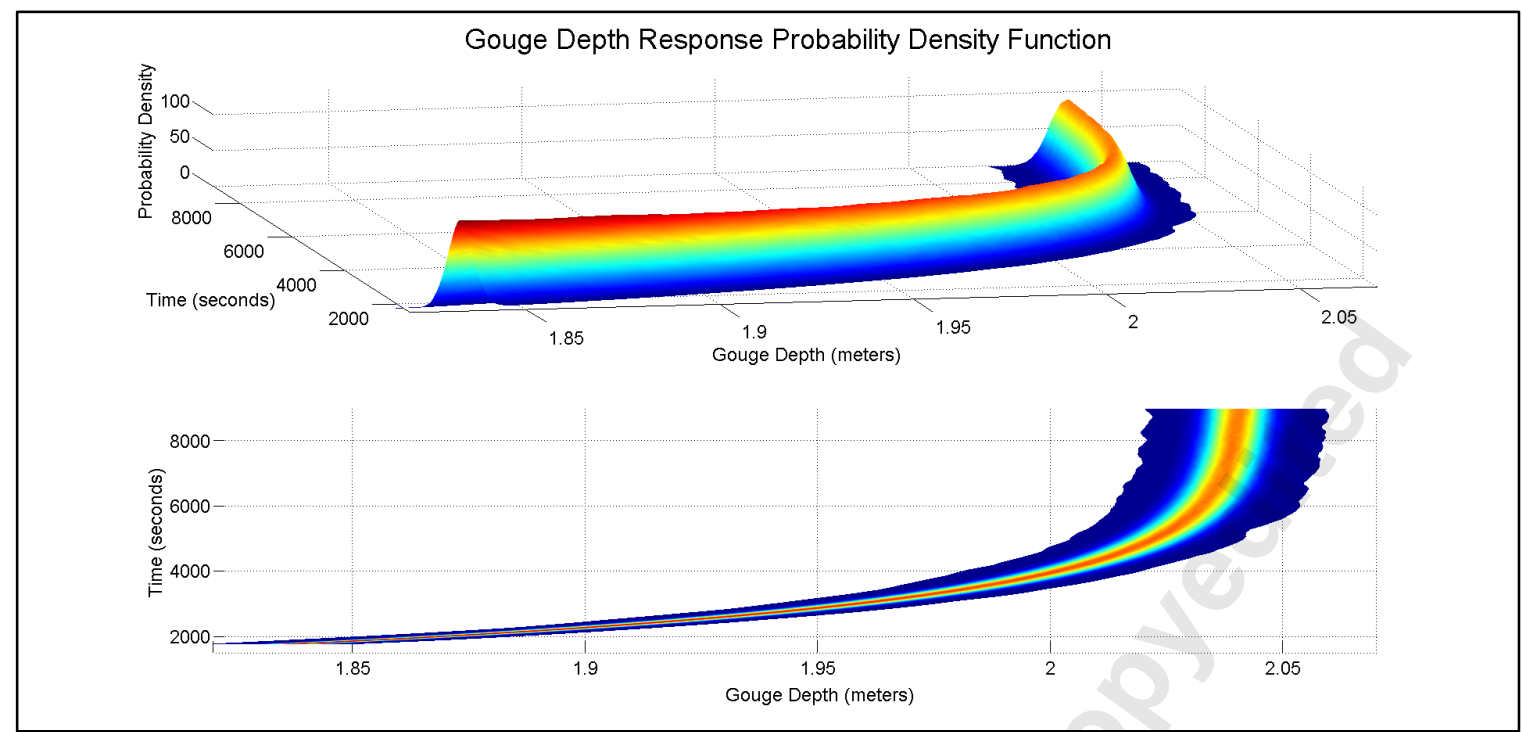

Fig.4. Gouge Depth Response PDF with Time (MCS) 


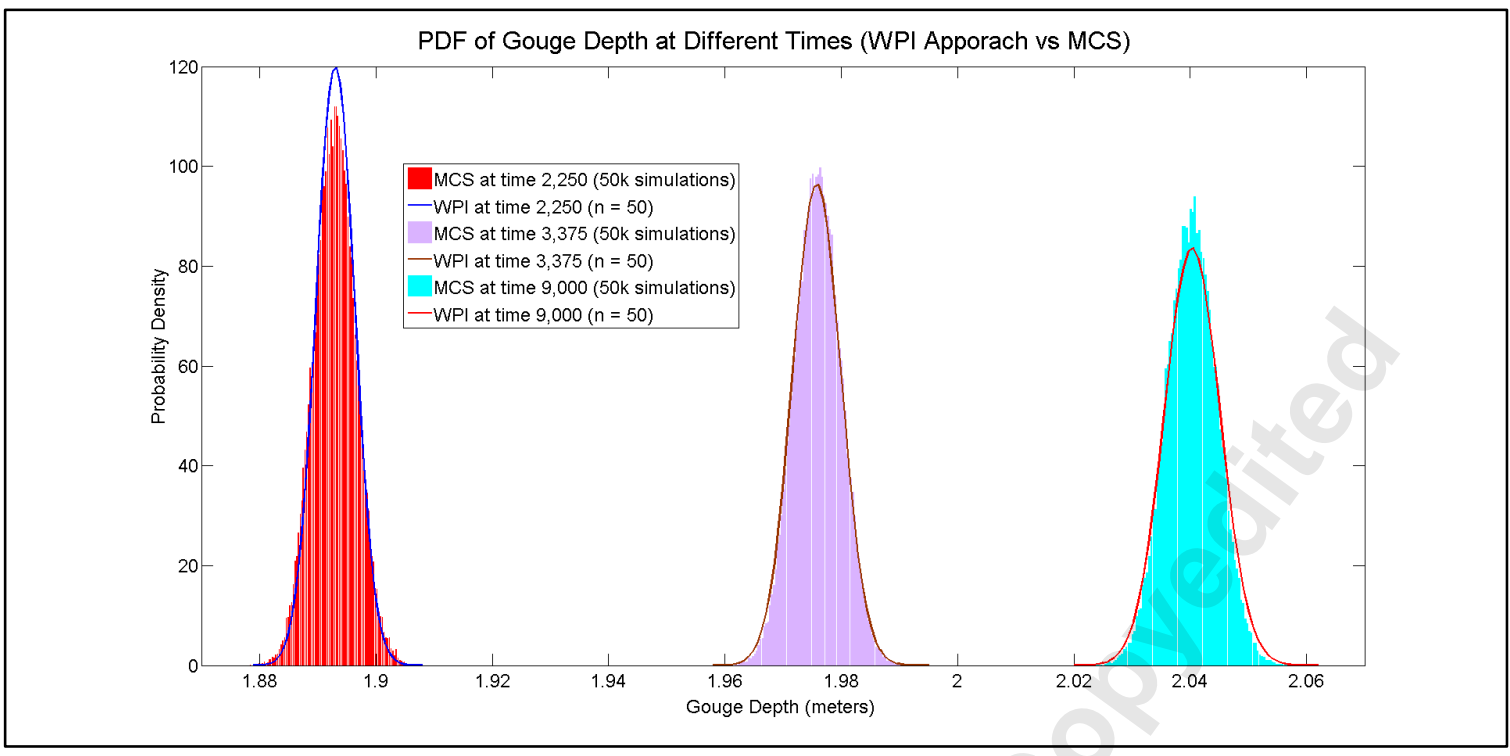

Fig.5. Gouge Depth Response PDF at Different Time Instances (WPI Approach vs. MCS) 


\begin{tabular}{|l|c|}
\hline Parameter & Value \\
\hline Berg mass, $\mathrm{m}$ & $20 \cdot 10^{9} \mathrm{~kg}$ \\
\hline Drag coefficient, A & $10^{-3} \mathrm{~m}^{-1}$ \\
\hline $\begin{array}{l}\text { Soil resistance } \\
\text { coefficient }^{1}, \mathrm{~B}\end{array}$ & $6 \cdot 10^{-9} \mathrm{~m}^{-1} \mathrm{~s}^{-2}$ \\
\hline $\begin{array}{l}\text { Mean sea current } \\
\text { velocity, } \mathrm{v}\end{array}$ & $0.5 \frac{\mathrm{m}}{\mathrm{s}}$ \\
\hline
\end{tabular}

${ }^{1}$ The value of $B$ considers a soil with relatively small cohesion, submerged unit weight of $2 \mathrm{kN} / \mathrm{m}^{3}$, bottom slope of $1 / 100$ and gouge width of 30 meters (e.g. 0).

Table 1. Inputs used in Numerical Example 